\title{
CHAIN TRANSITIVITY AND ROTATION SHADOWING FOR ANNULUS HOMEOMORPHISMS
}

\author{
FERNANDA BOTELHO AND LIANG CHEN
}

(Communicated by Charles Pugh)

\begin{abstract}
We present a relation between the rotation of chain transitive sets and the rotation shadowing for annulus homeomorphisms isotopic to identity.
\end{abstract}

\section{INTRODUCTION}

The concept of rotation shadowing was introduced by Barge and Swanson in [BS]. They conjectured that the rotation shadowing property is generic for degree one annulus homeomorphisms. Roughly, rotation shadowing means that for small $\delta>0$ the rotation averages along a $\delta$-pseudo-orbit can be uniformly approximated by the rotation averages along a true orbit; see $\S 2$ for the precise definition. In practice, rotation shadowing reveals whether computer calculations of rotation numbers are accurate.

In this paper we study the relationship between rotation shadowing and rotations of chain transitive sets for annulus homeomorphisms. Our main result is the following

Theorem. Suppose $\mathbf{A}$ is a compact annulus and $f: \mathbf{A} \rightarrow \mathbf{A}$ is a homeomorphism isotopic to identity. The rotation number is well defined on every chain transitive set in $\mathbf{A}$ if and only if $f$ has the rotation shadowing property and each point of A has a well-defined rotation number.

If the rotation set of an annulus homeomorphism is nowhere dense, [Sw] proves that the rotation number is well defined and varies continuously over chain recurrent sets in $\mathbf{A}$. In such a case, the results of [ $\mathrm{Fr}, \mathrm{Ha}$ ] show that the rotation number is also well defined on every chain transitive set (see [BC]). Consequently, the theorem implies that, under the same assumption, the annulus homeomorphism has the rotation shadowing property.

\section{Definitions}

Let $d$ be the Euclidean metric in a compact annulus $\mathbf{A}, \widetilde{\mathbf{A}}=\mathbf{R} \times[0,1]$ be the universal covering space of $\mathbf{A}$, and $p$ be the standard covering map. If $f$

Received by the editors December 27, 1991.

1991 Mathematics Subject Classification. Primary 34C35; Secondary 54H20.

Key words and phrases. Chain transitivity, shadowing, pseudo-orbit, rotation set, annulus homeomorphisms. 
is a map in $\mathbf{A}$, then let $F$ be a lift of $f$ to $\tilde{\mathbf{A}}$. We write $\tilde{x}_{1}=\pi_{1}(\tilde{x})$ and $F_{1}(\tilde{x})=\pi_{1}[F(\tilde{x})]$, where $\pi_{1}$ is the natural projection onto the first coordinate.

Given $x \in \mathbf{A}$, the rotation sequence $\left\{\theta_{n}(x)\right\}_{n \geq 1}$ of $x$ is defined to be

$$
\theta_{n}(x)=\frac{F_{1}^{n}(\tilde{x})-\tilde{x}_{1}}{n} .
$$

The rotation interval of $x$ is defined by

$$
\rho(x)=\left[\liminf _{n \rightarrow \infty} \theta_{n}(x), \limsup _{n \rightarrow \infty} \theta_{n}(x)\right] .
$$

The union of all rotation intervals is called the rotation set of $f$, denoted by $\rho(f)$. In particular, $x$ is said to have a well-defined rotation number if its rotation interval consists of a single point, also denoted by $\rho(x)$. If $S \subset \mathbf{R}$ is bounded, then $\langle S\rangle$ and $|S|$ will represent the convex hull of $S$ and the length of $\langle S\rangle$, respectively.

Given $\delta>0$, a sequence $\left\{z_{n}\right\}$ of points in $\mathbf{A}$ is called a $\delta$-pseudo-orbit of $f$ if, for all $n \geq 0$,

$$
d\left(z_{n+1}, f\left(z_{n}\right)\right)<\delta
$$

(see [Bow]). A $\delta$-pseudo-orbit of finite length is referred to as a $\delta$-chain (see [Co]). If $\left\{z_{n}\right\}$ is a $\delta$-pseudo-orbit of $f$, there exist lifts $\left\{\tilde{z}_{n}\right\}$ of $\left\{z_{n}\right\}$ such that $p\left(\tilde{z}_{n}\right)=z_{n}$ and $\left|F_{1}\left(\tilde{z}_{n}\right)-\pi_{1}\left(\tilde{z}_{n+1}\right)\right|<\delta$. The pseudo-rotation interval of $\left\{z_{n}\right\}$ is defined to be

$$
\rho\left\{\tilde{z}_{n}\right\}=\left[\liminf _{n \rightarrow \infty} \frac{\pi_{1}\left(\tilde{z}_{n}\right)}{n}, \limsup _{n \rightarrow \infty} \frac{\pi_{1}\left(\tilde{z}_{n}\right)}{n}\right] .
$$

Let $\rho(F, \delta)$ denote the union of all pseudo-rotation intervals associated with $\delta$-pseudo-orbits of $F$. The pseudo-rotation set of $F$ is defined by

$$
\rho_{\psi}(F)=\bigcap_{\delta>0} \rho(F, \delta),
$$

and the pseudo-rotation set of $f$ is given by $\rho_{\psi}(f) \equiv \rho_{\psi}(F)(\bmod 1)$.

For a set $\Lambda, \omega(\Lambda)$ denotes the union of the $\omega$-limit sets of points in $\Lambda$. If $\Lambda \subset \mathbf{A}$ is invariant under $f$, let $\rho\left(\left.f\right|_{\Lambda}\right)$ (resp. $\rho_{\psi}\left(\left.f\right|_{\Lambda}\right)$ ) be the rotation set of $\Lambda$ (resp. the pseudo-rotation set of $\Lambda$ ). The rotation number is well defined on $\Lambda$ if $\rho\left(\left.f\right|_{\Lambda}\right)$ consists of a single number.

The following definition is due to Barge and Swanson [BS]. An annulus map $f$ is said to have the rotation shadowing property if for any $\varepsilon>0$ we can find $\delta>0$ such that for every $\delta$-pseudo-orbit $\left\{z_{k}\right\}$ there exists a point $z$ in $\mathbf{A}$ satisfying

$$
\limsup _{k \rightarrow \infty} \frac{\left|\pi_{1}\left(\tilde{z}_{k}\right)-F_{1}^{k}(\tilde{z})\right|}{k}<\varepsilon .
$$

Correspondingly, $z$ is said to $\delta$-rotationally shadow $\left\{z_{k}\right\}$. It was shown in [BS] that every degree one endomorphism of the circle has the rotation shadowing property.

A set $\Lambda \subset \mathbf{A}$ is called $\alpha$-invariant if $d(f(x), \Lambda)<\alpha$ for all $x \in \Lambda . \quad \Lambda$ is $\alpha$-chain transitive if for every pair of points in $\Lambda$ there exists an $\alpha$-chain from the first element of the pair to the second one. For an $\alpha$-invariant and $\alpha$-chain transitive set $\Lambda, \rho_{\psi}\left(\left.f\right|_{\Lambda}, \alpha\right)$ is defined to be the union of the rotation 
intervals of all $\alpha$-pseudo-orbits in $\Lambda$. A set $\Lambda$ is chain transitive if $\Lambda$ is $\alpha$-chain transitive for all $\alpha>0$. Given a sequence of sets $X_{n}$, let

$$
\limsup _{n \rightarrow \infty} X_{n}=\bigcap_{N=1}^{\infty} \mathrm{cl}\left(\bigcup_{n \geq N}^{\infty} X_{n}\right) \text {. }
$$

\section{Proof of the theorem}

Let $f: \mathbf{A} \rightarrow \mathbf{A}$ be a homeomorphism isotopic to identity.

Lemma 1. If $\Lambda \subset \mathbf{A}$ is a compact invariant set, then

$$
\left\langle\rho_{\psi}\left(\left.f\right|_{\Lambda}\right)\right\rangle=\left\langle\rho\left(\left.f\right|_{\Lambda}\right)\right\rangle \text {. }
$$

If $\Lambda$ is replaced by the entire annulus, then $\rho_{\psi}(f)=\rho(f)$.

Proof. The first assertion is Lemma 3.4 in [BS]. The second is Theorem 3.2 of [BS] together with Handel's proof in [Ha] that the rotation set is always closed.

The next lemma is Lemma 3.9 of [BS].

Lemma 2. If $\Lambda \subset \mathbf{A}$ is compact, invariant, and chain transitive, then for each $\alpha>0$ there exists an $\alpha$-pseudo-orbit $\left\{z_{n}\right\}$ such that $\rho\left\{z_{n}\right\}=\rho_{\psi}\left(\left.f\right|_{\Lambda}\right)$.

Lemma 3. Suppose $\left\{\alpha_{m}\right\}$ is a sequence of positive numbers converging to 0 . If $\left\{\Lambda_{m}\right\}$ is a sequence of compact $\alpha_{m}$-invariant and $\alpha_{m}$-chain transitive sets in A which converges in the Hausdorff topology to the set $\Lambda$, then $\Lambda$ is compact invariant, chain transitive, and

$$
\rho_{\psi}\left(\left.f\right|_{\Lambda}\right) \supset \limsup _{m \rightarrow \infty} \rho_{\psi}\left(\left.f\right|_{\Lambda_{m}}, \alpha_{m}\right) .
$$

Proof. The invariance and chain transitivity are straightforward, and we leave them to the reader to verify (see $[B S]$ ).

If $\varepsilon>0$ we claim there is an $N$ such that, for $n>N, \rho_{\psi}\left(\left.f\right|_{\Lambda_{n}}, \alpha_{n}\right) \subset$ $\rho_{\psi}\left(\left.f\right|_{\Lambda}, \varepsilon\right)$. By the continuity of $F$ there exists $\eta \leq \varepsilon / 3$ such that $d(\tilde{z}, \tilde{w})<\eta$ implies that $d(F(\tilde{z}), F(\tilde{w}))<\varepsilon / 3$. We may suppose that $N$ is large enough, so that $d(w, \Lambda)<\eta$ and $\alpha_{n}<\varepsilon / 3$ for all $w \in \Lambda_{n}$ and $n \geq N$. For such $n$, suppose that $\left\{\tilde{w}_{k}\right\}$ is an $\alpha_{n}$-pseudo-orbit in $\tilde{\Lambda}_{n}$. If elements $\tilde{z}_{k} \in \tilde{\Lambda}$ are chosen with $d\left(\tilde{z}_{k}, \tilde{w}_{k}\right)<\eta$, then by the triangle inequality, $d\left(F\left(\tilde{z}_{k}\right), \tilde{z}_{k+1}\right)<\varepsilon$ for $k=0,1, \ldots$. Hence, $\left\{\tilde{z}_{k}\right\}$ is an $\varepsilon$-pseudo-orbit in $\widetilde{\Lambda}$ having the property that $d\left(\tilde{z}_{k}, \tilde{w}_{k}\right)<\eta$ for all $k \geq 0$. Evidently, $\rho\left\{\tilde{z}_{k}\right\}=\rho\left\{\tilde{w}_{k}\right\}$, and the claim is true. Now since

$$
\limsup _{n \rightarrow \infty} \rho_{\psi}\left(\left.f\right|_{\Lambda_{m}}, \alpha_{m}\right) \subset \bigcap_{\varepsilon>0} \rho\left(\left.f\right|_{\Lambda}, \varepsilon\right)=\rho_{\psi}\left(\left.f\right|_{\Lambda}\right),
$$

the lemma follows.

Proof of the theorem. Assume $f$ has the rotation shadowing property and that every orbit has a well-defined rotation number. If there exists a chain transitive set in $\mathbf{A}$ that admits two different rotation numbers $\{r, s\}$, then Lemma 2 implies that for any $\alpha>0$ there exists an $\alpha$-pseudo-orbit whose rotation set contains the interval $\langle\{r, s\}\rangle$. Since $f$ has the rotation shadowing property, 
for small enough $\alpha,\left\{z_{n}\right\}$ is $\frac{1}{3}|r-s|$-rotationally shadowed by some true orbit in A. It is clear that this true orbit cannot have a well-defined rotation number.

Suppose that every chain transitive subset of $\mathbf{A}$ has trivial rotation. If some point $z$ has a nondegenerate rotation interval, then $\omega(z)$ has nontrivial rotation (see [Boy, Lemma 1.1]). This would contradict the assumption because $\omega(z)$ is chain transitive.

Assume that the rotation shadowing fails to hold. We claim there exists a number $a>0$ such that for all $\delta>0$ there are $\delta$-pseudo-orbits $\left\{z_{n}(\delta)\right\}$ with the length of $\rho\left\{z_{n}(\delta)\right\}>a$. Otherwise, as $\delta \rightarrow 0$, the length of $\rho\left\{z_{n}(\delta)\right\} \rightarrow 0$ for all $\delta$-pseudo-orbits $\left\{z_{n}(\delta)\right\}$. In the latter case, given $\varepsilon>0$ for small $\delta>0$, we have $\rho\left\{z_{n}(\delta)\right\} \subset\left(\rho_{0}-\varepsilon, \rho_{0}+\varepsilon\right)$ for some $\rho_{0} \in \rho_{\psi}(f)$. By Lemma 1, $\rho_{0} \in \rho(f)=\rho_{\psi}(f)$, and some true orbit has rotation number $\rho_{0}$. This true orbit $\varepsilon$-rotationally shadows the pseudo-orbit $\{z(\delta)\}$.

To complete our proof we construct a sequence of subsets $\left\{\Lambda_{m}\right\}$ as in Lemma 3, so $\Lambda=\lim \sup _{m} \Lambda_{m}$ is chain transitive, and $\rho(\Lambda)$ has length at least $a>0$. We claim that for each $m>0$ there exists a $\frac{2}{m}$-invariant and $\frac{2}{m}$-chain transitive set $\Lambda_{m}$ such that the rotation set of $\Lambda_{m}$ has length at least $a>0$. For $m>0$ define

$$
\Lambda_{m}=\bigcap_{N=1}^{\infty} \operatorname{cl}\left(\left\{z_{n}\left(\frac{1}{m}\right): n \geq N\right\}\right) .
$$

For simplicity write $z_{n}=z_{n}(\delta) . \quad \Lambda_{m}$ is $\frac{3}{2 m}$-invariant. In fact, there exists $\eta>0$ such that $d(w, z)<\eta$ implies $d(f(w), f(z))<\frac{1}{2 m}$. For every $w \in \Lambda_{m}$ there exists $k$ such that $d\left(w, z_{k}\right)<\eta$. Then $d\left(f(w), z_{k+1}\right) \leq$ $d\left(f(w), f\left(z_{k}\right)\right)+d\left(f\left(z_{k}\right), z_{k+1}\right) \leq \frac{3}{2 m}$.

For any two points $x$ and $y$ in $\Lambda_{m}$, since $\Lambda_{m}$ is $\frac{3}{2 m}$-invariant, there exists $k, l$ such that $l>k>0, d\left(f(x), z_{k}\right)<\frac{2}{m}$, and $d\left(y, z_{l}\right)<\frac{1}{m}$. Define $w_{0}=x, w_{j}=z_{k+j-1}$ for $j=1, \ldots, l-k$, and $w_{l-k+1}=y$. Then $\left\{w_{j}\right\}$ is a $\frac{2}{m}$-chain from $x$ to $y$. Similarly, there is a $\frac{2}{m}$-chain from $y$ to $z$.

Choosing a subsequence, if necessary, we may suppose that $\Lambda=\lim _{m \rightarrow \infty} \Lambda_{m}$ exists in the Hausdorff topology. By Lemma $3, \Lambda$ is chain transitive, and the rotation set of $\left.f\right|_{\Lambda}$ contains an interval of length at least $a>0$, contradicting our assumption.

\section{ACKNOWLEDGMENT}

The authors are grateful for the referee's suggestions and comments that substantially shortened and simplified the proof of the theorem.

\section{REFERENCES}

[Bow] R. Bowen, On Axiom A diffeomorphisms, CBMS Regional Conf. Ser. in Math., vol. 35, SIAM, Philadelphia, PA, 1978.

[Boy] P. L. Boyland, Rotation sets and Morse decompositions in twist maps, Ergodic Theory Dynamical Systems 8 * (1988), 33-61.

[BC] F. Botelho and L. Chen, On the rotation shadowing property for annulus maps, Lecture Notes in Pure and Appl. Math., Marcel Dekker, New York (to appear).

[BS] M. Barge and R. Swanson, Rotation shadowing properties of circle and annulus maps, Ergodic Theory Dynamical Systems 8 (1988), 509-521. 
[Co] C. Conley, Isolated invariant sets and the Morse Index, CBMS Regional Conf. Ser. in Math., vol. 38, SIAM, Philadelphia, PA, 1978.

[Fr] J. Franks, Recurrence and fixed points of surface homeomorphisms, Ergodic Theory Dynamical Systems 8 * (1988), 99-107.

[Ha] M. Handel, The rotation set of a homeomorphism of the annulus is closed, Comm. Math. Phys. 127 (1990), 339-349.

[Sw] R. Swanson, Periodic orbits and the continuity of rotation numbers, Proc. Amer. Math. Soc. 117 (1993), 269-273.

Department of Mathematics, Memphis State University, Memphis, Tennessee 38152

E-mail address: Botelhof@hermes.MSCI.memst.edu

Department of Mathematics, Tufts University, Medford, Massachusetts 02155 